\title{
Cortical Low-Frequency Power and Progressive Phase Synchrony Precede Successful Memory Encoding
}

\author{
Rafi U. Haque, ${ }^{1}$ John H. Wittig, Jr., ${ }^{1}$ Srikanth R. Damera, ${ }^{1}{ }^{\circledR S}$ Sara K. Inati, ${ }^{2}$ and Kareem A. Zaghloul ${ }^{1}$ \\ ${ }^{1}$ Surgical Neurology Branch and ${ }^{2}$ Office of the Clinical Director, National Institute of Neurological Disorders and Stroke, National Institutes of Health, \\ Bethesda, Maryland 20892
}

\begin{abstract}
Neural activity preceding an event can influence subsequent memory formation, yet the precise cortical dynamics underlying this activity and the associated cognitive states remain unknown. We investigate these questions here by examining intracranial EEG recordings as 28 participants with electrodes placed for seizure monitoring participated in a verbal paired-associates memory task. We found that, preceding successfully remembered word pairs, an orientation cue triggered a low-frequency $2-4 \mathrm{~Hz}$ phase reset in the right temporoparietal junction with concurrent increases in low-frequency power across cortical regions that included the prefrontal cortex and left temporal lobe. Regions that exhibited a significant increase in 2-4 Hz power were functionally bound together through progressive low-frequency $2-4 \mathrm{~Hz}$ phase synchrony. Our data suggest that the interaction between power and phase synchrony reflects the engagement of attentional networks that in large part determine the extent to which memories are successfully encoded.
\end{abstract}

Key words: attention; episodic memory; phase synchrony

\section{Significance Statement}

Here we investigate the spatiotemporal cortical dynamics that precede successful memory encoding. Using intracranial EEG, we observed significant changes in oscillatory power, intertrial phase consistency, and pairwise phase synchrony that predict successful encoding. Our data suggest that the interaction between power and phase synchrony reflects the engagement of attentional networks that in large part determine the extent to which memories are successfully encoded.

\section{Introduction}

Successful memory formation relies on a complex interaction of various cognitive processes (Sekuler and Kahana, 2007). In the study of human memory, a number of specific patterns of neural activity linked to successful memory formation have been observed after item presentation (Staudigl and Hanslmayr, 2013; Yaffe et al., 2014). However, the functional state of the brain before item presentation may also affect the reliability of memory encoding. Such prestimulus influences may reflect optimal en-

\footnotetext{
Received Feb. 19, 2015; revised Aug. 4, 2015; accepted Aug. 12, 2015

Author contributions: R.U.H., J.H.W., and K.A.Z. designed research; R.U.H., S.R.D., and K.A.Z. performed research; S.K.I. contributed unpublished reagents/analytic tools; R.U.H. and K.A.Z. analyzed data; R.U.H., J.H.W., and K.A.Z. wrote the paper.

This work was supported by the Intramural Research Program of the National Institute for Neurological Disorders and Stroke. Data collection at the University of Pennsylvania and at Thomas Jefferson University was supported by National Institutes of Health Grants MH055687, MH061975, NS067316, and MH017168. We thank the clinical teams at the University of Pennsylvania Hospital and Thomas Jefferson Hospital for assistance in data collection. We are indebted to all patients who have selflessly volunteered their time to participate in this study. We thank John F. Burke for helpful and insightful comments on this manuscript.

The authors declare no competing financial interests.

Correspondence should be addressed to Dr. Kareem A. Zaghloul, Surgical Neurology Branch, National Institute of Neurological Disorders and Stroke, National Institutes of Health, Building 10, Room 3D20, 10 Center Drive, Bethesda, MD 20892-1414. E-mail: kareem.zaghloul@nih.gov.

DOI:10.1523/JNEUROSCI.0687-15.2015

Copyright $\odot 2015$ the authors $\quad 0270-6474 / 15 / 3513577-10 \$ 15.00 / 0$
}

coding states, attentional mechanisms, or motivation. Evidence for the role of prestimulus activity has emerged from recent studies demonstrating active modulation of frontal event-related potentials (Otten et al., 2006; Gruber and Otten, 2010), increases in hippocampal and parahippocampal fMRI BOLD activation (Adcock et al., 2006; Park and Rugg, 2010), and increases in hippocampal theta oscillatory power (Guderian et al., 2009; Fell et al., 2011) before item presentation.

Although these studies suggest that prestimulus neural activity contains relevant information for predicting later successful encoding, it is not clear what cognitive processes such prestimulus activity may represent. Are these changes specific to memory encoding regions, such as the medial temporal lobe (MTL), or do they reflect more distributed cortical processes, such as attention, that would benefit subsequent memory formation? Most evidence for prestimulus activity has focused on the hippocampus, a critical structure for the formation of contextually defined memories (Squire et al., 2004; Manns et al., 2007). However, only a limited number of studies have investigated prestimulus oscillatory activity in neocortical regions (Otten et al., 2006; Gruber and Otten, 2010; Addante et al., 2015). In addition, a number of questions regarding the precise spatiotemporal and network dynamics of pre-stimulus activity are unresolved. If prestimulus activity 
reflects engagement for an individual stimulus (Haynes et al., 2007), rather than a randomly fluctuating and generalized arousal state (Linkenkaer-Hansen et al., 2004), then the neural mechanisms underlying this activity should exhibit a well defined progression in time and space. Whether such engagement is mediated through changes in oscillatory power and functional connectivity between anatomic regions and which frequency bands specifically contribute to this process remain unknown.

We investigate these questions here by examining prestimulus changes in intracranial EEG (iEEG) recordings as patients with electrodes placed for seizure monitoring participated in a verbal pairedassociates memory task. We examine oscillatory power, intertrial phase consistency (ITPC), and phase synchrony in five frequency bands to explore neural mechanisms distributed across cortical regions that predict subsequent memory encoding. We find significant increases in low-frequency $2-4 \mathrm{~Hz}$ oscillatory power before the presentation of word pairs during successful encoding, and, leveraging the high temporal resolution afforded by iEEG, we investigate the temporal dynamics of these changes throughout the cortex. We then asked whether specific cortical regions demonstrating increases in spectral power before successful memory formation also exhibit increased pairwise phase synchrony. We tracked the temporal evolution of these interactions to identify evolving network dynamics that may reflect attentional processes that optimize the functional state of the brain for successful encoding.

\section{Materials and Methods}

Participants. Twenty-eight participants (17 males, 11 females; aged $33.1 \pm 2.64$ years) with medication-resistant epilepsy underwent a surgical procedure in which platinum recording contacts were implanted subdurally on the cortical surface and deep within the brain parenchyma. In each case, the clinical team determined the placement of the contacts so as to best localize epileptogenic regions. Data were collected at three different hospitals: (1) the Clinical Center at the National Institutes of Health (NIH; Bethesda, MD); (2) the Hospital of the University of Pennsylvania (UP; Philadelphia, PA); and (3) Thomas Jefferson University Hospital (TJ; Philadelphia, PA). The research protocol was approved by the institutional review board at each hospital, and informed consent was obtained from the participants and their guardians.

Paired-associates task. Each patient participated in a paired-associates task (Fig. 1A). Participants were asked to study a list of word pairs and then were later cued with one word from each pair, selected at random. Participants were instructed to vocalize the partner of each cue word. Lists were composed of four pairs of common nouns, chosen at random and without replacement from a pool of high-frequency nouns. Words were presented sequentially and appeared in capital letters at the center of the screen. Study word pairs were separated from their corresponding recall cue by a minimum lag of two study and/or test items. During the study period (encoding), each word pair was preceded by an orientation cue (a row of capital X letters) that appeared on the screen for $300 \mathrm{~ms}$, followed by a blank interstimulus interval (ISI) of $750 \mathrm{~ms}$ with a jitter of $75 \mathrm{~ms}$. Word pairs were then presented on the screen for $2500 \mathrm{~ms}$, followed by a blank ISI of $1500 \mathrm{~ms}$ with a jitter of $75 \mathrm{~ms}$. During the test period (retrieval), one randomly chosen word from each study pair was shown, and the participant was asked to recall the other word from the pair by vocalizing a response into a microphone. Each cue word was preceded by an orientation cue (a row of question marks) that appeared on the screen for $300 \mathrm{~ms}$, followed by a blank ISI of $750 \mathrm{~ms}$ with a $75 \mathrm{~ms}$ jitter. Cue words were then presented on the screen for $3000 \mathrm{~ms}$, followed by a blank ISI of $4500 \mathrm{~ms}$. Participants could vocalize their response any time during the recall period after cue presentation. Vocalizations were recorded digitally and then scored manually for analysis. Responses were designated as correct, intrusions, or passes. Passes were categorized when no vocalization was made or when the participant vocalized the word "pass." Intrusion and pass trials were designated as incorrect trials. A single experimental session contained up to 25 lists. We excluded all subjects with recall percentages below $15 \%$ from subsequent analyses.

$i E E G$ recordings. Data were collected in collaboration with three different hospitals. Although each hospital used the same general implantation procedures and data-acquisition techniques, our analysis had to account for technical details that varied by institution. iEEG data were recorded using a Nihon Kohden (NIH and TJ) or Nicolet (UP) EEG data acquisition system. Depending on the amplifier and the discretion of the clinical team, EEG signals were sampled at 1000 or $2000 \mathrm{~Hz}$. Signals were referenced to a common contact placed subcutaneously, on the scalp, on the mastoid process, or intracranially. All recorded traces were resampled at $1000 \mathrm{~Hz}$, and a fourth-order $2 \mathrm{~Hz}$ stop-band Butterworth notch filter was applied at $60 \mathrm{~Hz}$ to eliminate electrical line noise. The testing laptop sent $\pm 5 \mathrm{~V}$ digital pulses via an optical isolator into a pair of open lines on the clinical recording system to synchronize the electrophysiological recordings with behavioral events.

We collected electrophysiological data from a total of 2218 subdural and depth recording contacts (PMT Corporation; AdTech). Subdural contacts were arranged in both grid and strip configurations with an intercontact spacing of $10 \mathrm{~mm}$. Hippocampal depth electrodes (six to eight linearly arranged contacts) were placed in three patients. Contact localization was accomplished by coregistering the postoperative computed tomographies with the postoperative MRIs using both Functional MRI of the Brain (FMRIB) Software Library BET (for Brain Extraction Tool) and FLIRT (for FMRIB Linear Image Restoration Tool) software packages and mapped to both Montreal Neurological Institute (MNI) and Talairach space using an indirect stereotactic technique and OsiriX Imaging Software (Digital Imaging and Communications in Medicine viewer package). The resulting contact locations were subsequently projected to the cortical surface of an MNI N27 standard brain (Dykstra et al., 2012). Preoperative MRIs were used when postoperative MR images were not available.

We analyzed iEEG data using bipolar referencing to reduce volume conduction and confounding interactions between adjacent electrodes. 
We defined the bipolar montage in our dataset based on the geometry of iEEG electrode arrangements. For every grid, strip, and depth probe, we isolated all pairs of contacts that were positioned immediately adjacent to one another; bipolar signals were then found by differencing the signals between each pair. The resulting bipolar signals were treated as new virtual electrodes (henceforth referred to as electrodes), originating from the midpoint between each contact pair. All subsequent analyses were performed using these derived bipolar signals. In total, our dataset consisted of 2329 electrodes (918 left hemispheric, 1411 right hemispheric; Fig. 1B).

Spectral power. To quantify changes in spectral power during the prestimulus encoding period, we bandpass filtered the iEEG signal for every electrode in five frequency bands: (1) $2-4 \mathrm{~Hz}$ (delta/low theta); (2) 4-8 $\mathrm{Hz}$ (theta); (3) $9-15 \mathrm{~Hz}$ (alpha); (4) $13-25 \mathrm{~Hz}$ (beta); and (5) $45-95 \mathrm{~Hz}$ (gamma). We used a Hilbert transform to obtain a continuous-time complex valued representation of the filtered signal. We squared and log transformed the magnitude of the continuous-time complex valued Hilbert transform to generate a continuous measure of instantaneous power. We separately aligned each encoding event to word presentation ( $1500 \mathrm{~ms}$ before to $500 \mathrm{~ms}$ after) and to the presentation of the orientation cue (750 ms before to $1650 \mathrm{~ms}$ after). We binned the continuous time transforms into $500 \mathrm{~ms}$ epochs spaced every $100 \mathrm{~ms}$ ( $80 \%$ overlap) and averaged the instantaneous power within each epoch. To account for changes in power across experimental sessions, we $z$ transformed power values separately for each frequency and session using the mean and SD of the average power of 500 randomly drawn $500 \mathrm{~ms}$ epochs from that session.

Phase synchrony and intertrial phase coherence. To obtain an estimate of inter-electrode phase synchrony, we first extracted the instantaneous phase of the complex valued Hilbert transform, $\phi(t, f)$, for every electrode and frequency band, $f$. For every frequency band, we calculated a phase-locking value $\left(\bar{R}_{p q}\right)$ between the continuous-phase series of two electrodes, $\phi_{p}(t, f)$ and $\phi_{q}(t, f)$ (Lachaux et al., 1999):

$$
\bar{R}_{p q}(f)=\frac{1}{S}\left|\sum_{t=1}^{S} e^{i\left(\phi_{p}(t, f)-\phi_{q}(t, f)\right)}\right|,
$$

where $S$ is the total number of samples from all trials in the temporal epoch of interest. We separately obtained a phase-locking value for correct and incorrect trials for every temporal epoch, frequency band, and pair of electrodes.

To obtain an estimate of ITPC, we again used the instantaneous phase of the complex valued Hilbert transform, $\phi_{p}(t, f)$, for every electrode and frequency band, $f$. In this case, for every frequency band, we calculated phase consistency $\left(\bar{R}_{p}\right)$ of a single electrode across trials at each sample of the instantaneous-phase signal (Lachaux et al., 1999):

$$
\bar{R}_{p}(f)=\frac{1}{N}\left|\sum_{t=1}^{N} e^{i\left(\phi_{p}(t, f)\right.}\right|
$$

where $N$ is total number of correct or incorrect trials. We calculated ITPC as the average $\bar{R}_{p}(f)$ within each temporal epoch for each frequency band and electrode.

For phase synchrony and ITPC, comparisons between correct and incorrect trials can be biased by mismatched trial counts. To compare values between conditions in an unbiased manner, we used a bootstrap procedure (Staudigl et al., 2015). We randomly subsampled trials from the condition with the larger number of trials to match the number of trials in the other condition. We repeated this procedure 500 times and calculated the phase synchrony or ITPC during each iteration. We assigned the average value of these iterations as the final value for the higher-trial-count condition and used this average bootstrapped value for comparison with the lower-trial-count condition.

Across-participant analysis in predefined anatomic regions. We investigated prestimulus encoding changes in predefined anatomic regions that have been implicated in vision (Leube et al., 2001), attention (Corbetta and Shulman, 2002; Corbetta et al., 2008), executive control (Smith and Jonides, 1999), and memory encoding (Burke et al., 2013). For each subject, we identified all electrodes within a visual perception region
[Brodmann area (BA) 17-BA 19], a frontal attention region (BA 8-BA 9), a parietal attention region (BA1, BA7, BA 39-BA 40), an executive control region (BA 10-BA12), and an MTL region (BA 34-BA 37 and hippocampus depth electrodes). We assessed differences in spectral power between correct and incorrect trials within these predefined regions using a permutation procedure identical to what we used for individual regions of interest (ROIs) (see below, Statistical Analyses of Individual ROIs).

Spatial ROIs for whole-brain analysis. With iEEG, the precise placement of electrodes is different for each participant, which limits our ability to examine spatially resolved effects across subjects. We overcame this limitation by spatially smoothing electrode effects using 760 spatial ROIs that were spaced evenly every $9.98 \pm 0.02 \mathrm{~mm}$ on the cortical surface of an MNI N27 standard brain. For each participant, we averaged the measured responses from all electrodes that were within $12.5 \mathrm{~mm}$ of a given ROI. Most electrodes contributed to more than one ROI, and most ROIs included either zero or more than one electrode per subject. When performing whole-brain analyses across participants, only ROIs that contained electrodes from five or more participants were evaluated.

To visualize the whole-brain results, we rendered cortical topographic plots based on the values computed at each ROI. For each vertex of the $3 \mathrm{D}$ rendered image of the standard brain, we computed the average value of all ROIs that were within $12.5 \mathrm{~mm}$ of that vertex. For topographic plots demonstrating significant differences between trials types, all colored regions, independent of color intensity, indicate two-tailed significance at the $p<0.05$ level as determined by the nonparametric clusteringbased procedure described below.

Statistical analyses of individual ROIs. For each participant, we calculated the trial-averaged $z$-scored spectral power and ITPC at each electrode. We did this separately for correct and incorrect trials at each frequency band and temporal epoch. We then spatially smoothed the power and ITPC values of each electrode by converting to ROIs as described above. For each participant, we were left with an average correct and an average incorrect value in each ROI at each temporal epoch and frequency band. Phase synchrony is a pairwise measure between two ROIs. For phase synchrony, we defined one set of ROIs as a seed region and then calculated phase synchrony between that seed region and all other ROIs across the cortical surface at each temporal epoch and frequency band.

We performed a random-effects statistical analysis at each ROI across participants. Our null hypothesis was that, across participants, the brain region represented by each ROI showed no difference in power, ITPC, or phase synchrony during correct versus incorrect trials. We tested this hypothesis using a nonparametric permutation procedure in which the participant is the unit of observation (Maris and Oostenveld, 2007). For each ROI, a minimum of five participants contributed trial-averaged correct and incorrect values. We computed the true mean difference between correct and incorrect trials across participants using these values. We then randomly permuted the participant-specific averages (correct vs incorrect) within each participant and recomputed the mean difference. For $n$ participants, this results in an empiric distribution of $2^{n}$ possible mean differences that are all equally probable under the null hypothesis. We generated the empiric distribution from a minimum of 32 ( $n=5$ participants) and a maximum of 1000 permutations for every ROI. We compared the true mean difference in each ROI to the mean and $\mathrm{SD}$ of this empiric distribution to generate an across-participant $z$ score for each ROI. We converted each $z$ score to a $p$ value using a normal cumulative distribution function. This $p$ value represents the likelihood that the true mean difference at an individual ROI represents a departure from the null hypothesis. However, this $p$ value for each individual ROI does not take into account the multiple comparisons that are made in space (across ROIs) or time (across epochs) and therefore is not reported in the text.

Space-time correction for multiple comparisons. To correct for multiple comparisons across ROIs (space) and temporal epochs (time), we used a nonparametric cluster correction method that incorporates biophysically motivated constraints into the cluster test statistic (Maris and Oostenveld, 2007). Specifically, the method assumes that a true effect at any given ROI is likely to be observed across consecutive temporal epochs at 
that ROI because of temporal averaging and, similarly, that a true effect is likely to be observed at adjacent ROIs because of spatial averaging. The first step in defining space-time clusters is to threshold the acrossparticipant values derived from the statistical analysis of individual ROIs described above. We used a $z$-score threshold of 1.645 for each ROI in each temporal epoch, applied separately to positive and negative effects. A space-time cluster is defined as an ROI with two or more consecutive temporal epochs that surpassed this threshold and/or two or more adjacent ROIs (ROIs within $12.5 \mathrm{~mm}$ of each other) that surpassed this threshold at a particular epoch. For each identified cluster, we defined our cluster test statistic to be the sum of the $z$ scores for all ROIs within that space-time cluster.

Space-time clusters were also calculated for 1000 permutations of the participant-specific trial averages, and the maximum cluster test statistic of each permutation was used to create an empiric distribution for significance testing. For each permutation, the same set of participantspecific trial labels were used for all ROIs and time epochs so that the biophysically motivated constraint of spatiotemporal contiguity was expressed in the empiric distribution. We determined whether a true cluster test statistic was significant by comparing it with the empiric distribution of maximum cluster test statistics. In this manner, significant clusters can arise from large differences between correct and incorrect trials that extend over a small region of space and time or from smaller differences that persist over larger regions. Cluster test statistics with $p<0.05$ were considered significant and corrected for multiple comparisons.

Some significant clusters were spatially constrained to a specific brain area, but more often a single large cluster included multiple brain areas, each of which had significant differences at seemingly different temporal epochs. To examine the spatiotemporal evolution of changes in spectral power, ITPC, and phase synchrony, we partitioned large significant clusters into distinct brain regions. Hence, each partitioned region was a subset of ROIs from the larger cluster that had been corrected for multiple comparisons in space and time. Plots of temporal evolution for each partitioned region represent the average and SEM of the differences in ITPC, synchrony, and $z$-scored power across participants. Temporal epochs for each partitioned region were deemed significant, and corrected for multiple comparisons, if the larger cluster included at least one ROI from the partitioned region at that epoch.

\section{Results}

Twenty-eight participants with intracranial electrodes for seizure monitoring participated in a verbal paired-associates task (Fig. $1 A, B)$. Participants studied $216 \pm 19$ word pairs and successfully recalled $34.8 \pm 3.5 \%$ words with a mean response time of $1908 \pm$ $106 \mathrm{~ms}$. On $13.7 \pm 1.9 \%$ of trials, participants responded with an incorrect word (intrusions). The mean response time for intrusions was $2826 \pm 121 \mathrm{~ms}$. For the remaining study word pairs, participants either made no response to the cue word or vocalized the word "pass." Participants vocalized the word "pass" with a mean response time of $3467 \pm 179 \mathrm{~ms}$ (Fig. 1C). We compared the recall rate for pairs immediately after the presentation of successfully encoded pairs to the overall recall rate. We found no significant difference across participants $\left(t_{(54)}=0.34, p>0.05\right)$, indicating that word pairs were not remembered consecutively. A one-way ANOVA also revealed no significant effect of study-test lag on recall probability $\left(F_{(4,155)}=1.06, p=0.38\right)$.

\section{Prestimulus oscillatory power in the frontal lobe predicts subsequent memory}

The neural activity that precedes successful memory encoding may result from a number of mental processes, including attention and executive control. As such, we performed an acrossparticipant analysis in predefined anatomic regions (see Materials and Methods) to compare the difference in spectral power between correct and incorrect trials. We averaged power changes over the entire prestimulus period, from -1000 to $0 \mathrm{~ms}$ before word-pair presentation. We found significant increases during correct trials in low-frequency $2-4 \mathrm{~Hz}$ power in a predefined frontal attention region during the prestimulus period (Fig. 2A). We did not observe significant differences during the prestimulus period in a predefined parietal attention, executive control, visual perception, or MTL region. We examined the time course and frequency range of this effect in a single electrode on the inferior frontal gyrus of an individual participant. Correct trials were associated with significant increases in low-frequency $(2-7 \mathrm{~Hz})$ power and significant decreases in high-frequency (16$128 \mathrm{~Hz})$ power before word-pair presentation $(p<0.05$, permutation test; Fig. 2B).

\section{Spatiotemporal dynamics of low-frequency power predict subsequent memory}

To explore the spatiotemporal dynamics of prestimulus differences in spectral power with greater temporal and spatial resolution across subjects, we investigated these differences during overlapping $500 \mathrm{~ms}$ temporal epochs using finely spaced ROIs that covered the whole brain (see Materials and Methods). We identified contiguous clusters of ROIs in space and time that exhibited a significant difference in spectral power between correct and incorrect trials ( $p<0.05$, permutation procedure, corrected for multiple comparisons; see Materials and Methods). During correct trials, we observed significant increases in lowfrequency power that began in the right and left prefrontal cortex (PFC) and right temporoparietal junction (TPJ) 1300-800 ms before pair presentation and that ended in the left posterior temporal lobe by the time of word-pair presentation (Fig. 2C, right). Also during correct trials, we observed significant decreases in high-frequency power in the left PFC 1300 to $800 \mathrm{~ms}$ before pair presentation and the right inferior frontal lobe by the time of word-pair presentation (data not shown).

Our ROI-based whole-brain analysis identified multiple brain areas with significant differences in low-frequency power across participants. Across incrementing temporal epochs, the brain areas showing significant differences changed (Fig. $2 C$, right), suggesting that different anatomic regions exhibit increases in lowfrequency power with different time courses. To examine this, we identified all ROIs that showed significant increases in lowfrequency power at any time point and partitioned these ROIs into a right TPJ (BA 1 and BA 40), a left PFC (BA 8-BA 12), and a left temporal lobe region. Across participants, we found that significant increases in right TPJ low-frequency power occurred early during the prestimulus period (1250 to $750 \mathrm{~ms}$ before pair presentation; Fig. 2D). We also found that low-frequency power exhibited significant increases in the left PFC and left temporal lobe during most of the prestimulus period.

\section{Spatiotemporal dynamics of low-frequency power show task responsiveness}

Given that these changes occur after presentation of an orientation cue, we were also interested in understanding whether the prestimulus period showed changes in response to the cue independent of successful encoding. For each participant, we compared the average spectral power across all trials (in this case, combining correct and incorrect trials) after cue presentation, which occurs $1050 \pm 75$ ms before pair presentation, to a baseline period comprising $500 \mathrm{~ms}$ epochs sampled randomly throughout the session. We identified brain areas that exhibited a significant difference between the task and baseline $(p<0.05$, permutation procedure; see Materials and Methods). We found significant 

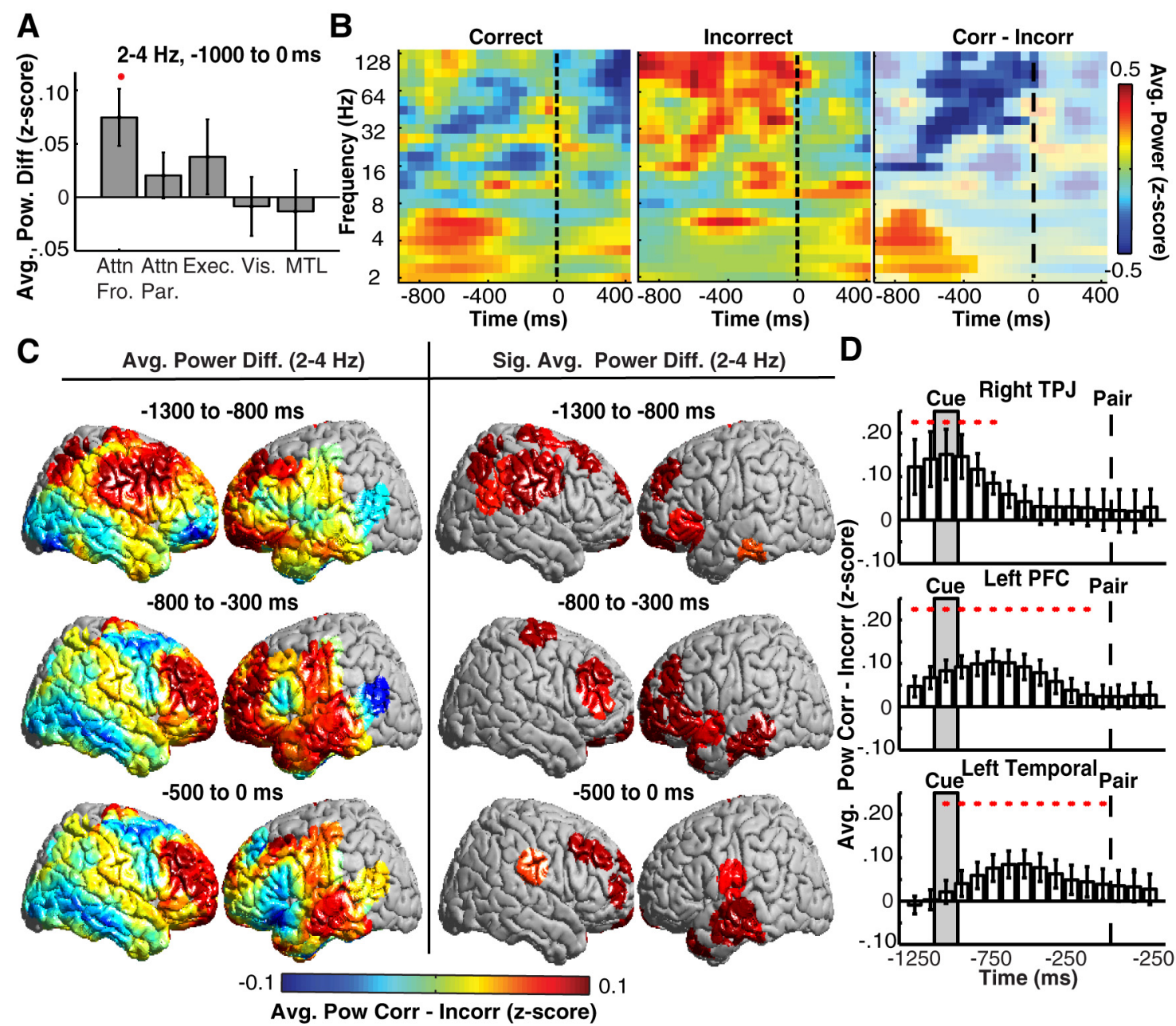

Figure 2. Low-frequency prestimulus power changes in space and time predict memory formation across participants. A, Prestimulus differences in low-frequency (2-4 Hz) power between correct and incorrect trials across participants in frontal attention (Attn Fro.), parietal attention (Attn Par.), executive control (Exec.), visual (Vis.), and MTL regions during the prestimulus period. Regions showing significantly greater power during correct trials are indicated with a red circle ( $p<0.05$, permutation procedure). $\boldsymbol{B}$, Exemplar prestimulus spectral power from a single electrode on the inferior frontal gyrus for correct (left) and incorrect (middle) trials and the difference between them (right). Significant differences between correct (Corr) and incorrect (Incorr) trials are unmasked. C, Prestimulus differences in low-frequency (2-4 Hz) power between correct and incorrect trials across the whole brain during three prestimulus temporal epochs (left). ROls exhibiting significant ( $p<0.05$, corrected for multiple comparisons) differences across participants are shown (right). $\boldsymbol{D}$, Time evolution of average power differences between correct and incorrect trials for the right TPJ, left PFC, and left temporal lobe regions that were identified using our whole-brain analysis (C). Significant temporal epochs are indicated with a red circle ( $p<0.05$, corrected for multiple comparisons in space and time). Word pair and cue onset are indicated by the dashed line and gray region, respectively. Error bars represent SEM across subjects.

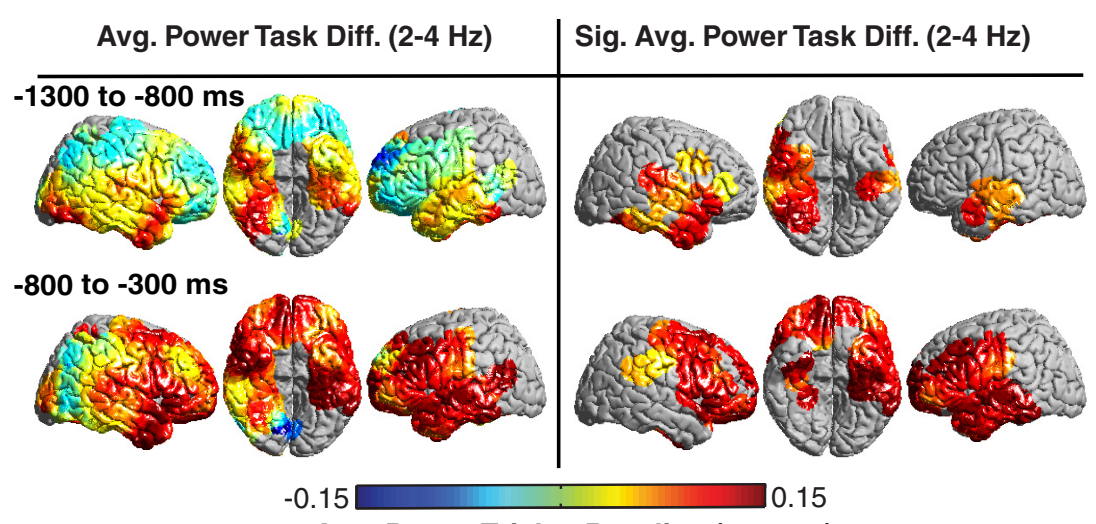

Avg. Power Trials - Baseline (z-score)

Figure 3. Low-frequency prestimulus power predicts task responsiveness across participants. Prestimulus differences in lowfrequency ( $2-4 \mathrm{~Hz}$ ) power between all trials and baseline (left). ROls exhibiting significant $(p<0.05$, corrected for multiple comparisons) differences across participants are shown (right). bilateral increases in low-frequency $(2-4$ $\mathrm{Hz}$ ) power that began in the inferior temporal lobes 1300 to $800 \mathrm{~ms}$ before pair presentation and extended to the parietal and frontal lobes 800 to $300 \mathrm{~ms}$ before pair presentation (Fig. 3, right). Notably, these task-responsive increases did not originate in left frontal regions that exhibited significant differences between correct and incorrect trials during the prestimulus period (Fig. 2C).

Phase reset marks the onset of memoryrelated power changes

Because the orientation cue in our task is presented $1050 \pm 75$ ms before pair presentation, we investigated whether the early increases in power are related to the orientation cue that is visually presented 
in the task. Previous studies have demonstrated that attention to visual cues may elicit a phase reset in the local field potential signal (Daitch et al., 2013). We timelocked our data to the orientation cue and investigated differences in ITPC between correct and incorrect trials. Using our ROI-based whole-brain analysis, we found significant increases in lowfrequency ITPC in correct compared with incorrect trials after cue presentation in the right TPJ, a right temporooccipital (TO) region, and a right temporal lobe region ( $p<0.05$, permutation procedure; Fig. $4 A$ ). We did not observe significant differences in ITPC in any other anatomic region or frequency band ( $p>0.05$, permutation procedure). We reexamined spectral power time-locked to the cue presentation and confirmed that a similar pattern of activation was observed when locked to pair presentation. The time course of cue-locked ITPC differences suggests that the right TPJ is one of the earliest sites of a phase reset that precedes successful memory encoding (Fig. 4B).

\section{Spatiotemporal dynamics of pairwise phase synchrony predict subsequent memory}

We investigated whether the widespread oscillatory power and phase effects reflect increased functional connectivity between brain regions. We defined two seed regions, the right TPJ and the left PFC, that exhibited significant increases in spectral power in the prestimulus period during correct encoding. For each seed region, we examined the differences in low-frequency pairwise phase synchrony across all brain ROIs for correct versus incorrect trials. During correct trials, the right TPJ exhibited significant increases in low-frequency phase synchrony with the right frontal lobe from the time of cue presentation to $950 \mathrm{~ms}$ after cue presentation $(p<0.05$, permutation procedure; Fig. $5 A$ ). Also during correct trials, the left PFC seed exhibited significant increases in low-frequency phase synchrony with the right frontal lobe and the left dorsolateral PFC at the time of cue presentation, followed by later increases in phase synchrony with regions in the left parietal and temporal lobes 550-1050 ms after cue presentation ( $p<0.05$, permutation procedure; Fig. $5 B)$.

We examined the time course of phase synchrony among brain areas deemed significant using our ROI-based whole-brain analysis. Specifically, we examined the temporal evolution of phase synchrony between four pairs of regions: (1) a right TPJ and right frontal region; (2) a left PFC and right frontal region; (3) a left PFC and left parietal region; and (4) a left PFC and left temporal region (Fig. $5 C$ ). The time course of phase synchrony increase appeared to be different for these four pairs of regions, with some areas synchronizing near the onset of the orientation cue, some synchronizing immediately before the word-pair presentation, and some synchronizing throughout the entire prestimulus period.

Because the temporal lobe, and particularly the MTL structures, have been implicated in a number of studies investigating prestimulus changes during memory encoding (Guderian et al.,
2009; Fell et al., 2011), we were also interested in understanding the changes in functional connectivity with this region. We created a new seed region comprising all left MTL ROIs and examined the differences in low-frequency phase synchrony across all brain regions for correct versus incorrect trials. During correct trials, we found significant increases in low-frequency phase synchrony with the left PFC and lateral temporal lobe $(p<0.05$, permutation procedure; Fig. $6 A$ ). The time course of these changes revealed significant increases in low-frequency phase synchrony between the left MTL and the left PFC 850-1350 ms after cue presentation, which coincides with when the word pair was presented.

The observed significant increases in pairwise phase synchrony across anatomic regions suggest a temporal progression of functional connections that precede successful encoding. To investigate this, we calculated the time at which the difference between correct and incorrect trials peaked for each pair of identified seed regions evaluated in Figures $5 C$ and $6 B$. Across participants, we found a significant effect of anatomic connections on the time of peak increase in pairwise synchrony $\left(F_{(3,18)}=3.87\right.$, $p=0.03$, repeated-measures ANOVA). A temporal progression of functional connections starts with the right TPJ and frontal cortex, involves the right and left frontal cortices, and ends with functional connections between the left PFC and the left parietal and temporal lobes (Fig. 6C). This procession starts at orientation cue onset and ends at the time of word-pair presentation during correct trials.

\section{Discussion}

Our data demonstrate that increases in low-frequency $2-4 \mathrm{~Hz}$ oscillatory power, ITPC, and phase synchrony arise before the 
A

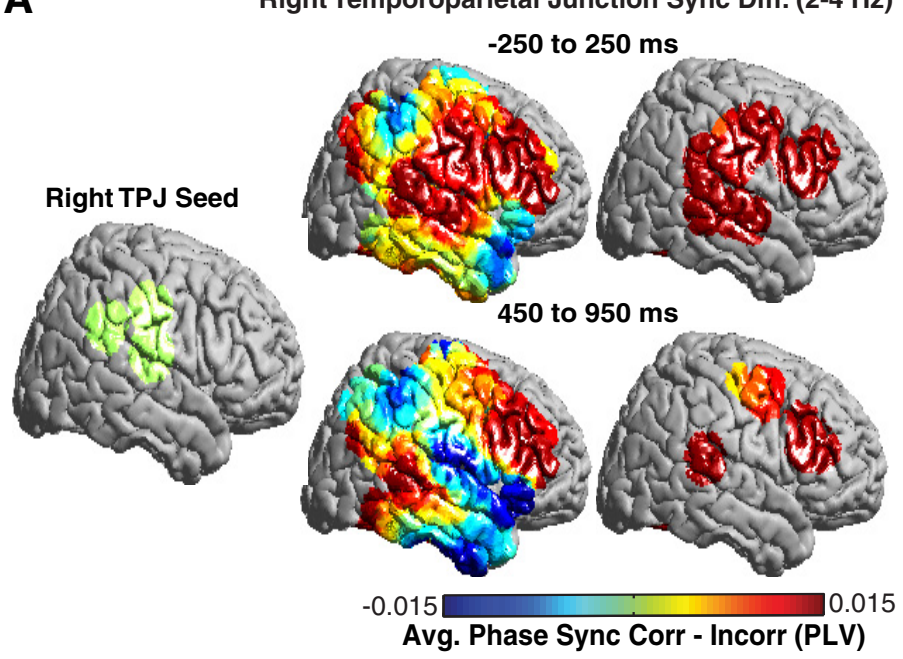

B

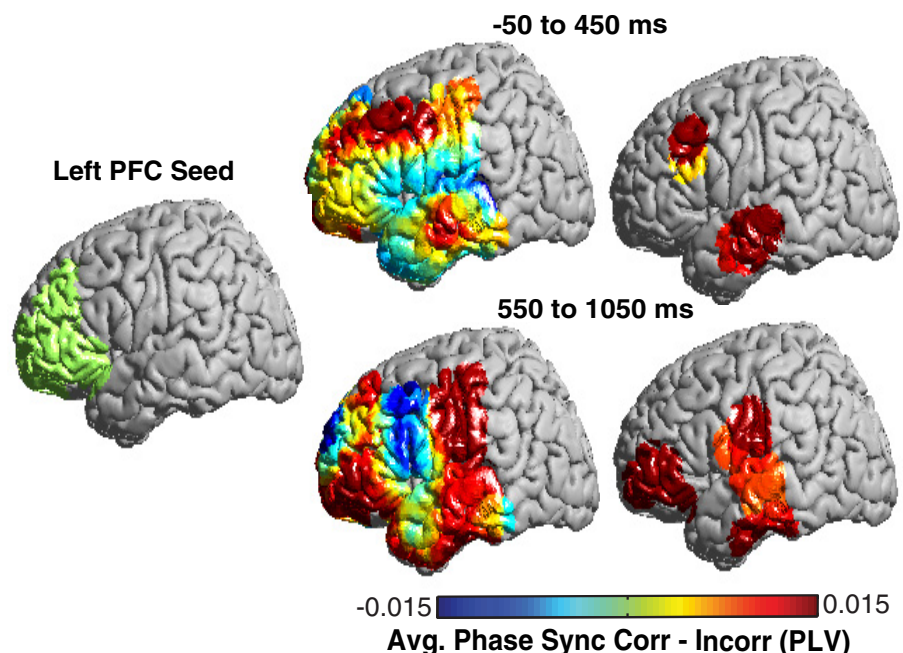

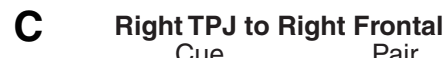
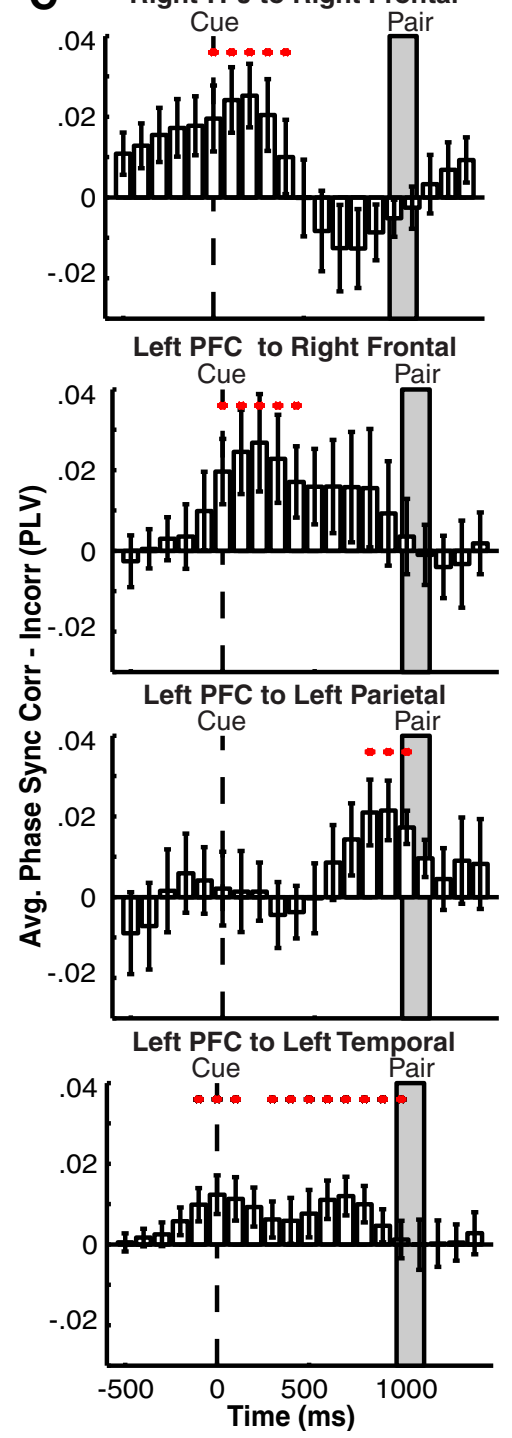

Figure 5. Prestimulus increases in low-frequency pairwise synchrony after cue presentation predicts successful memory encoding across participants. $\boldsymbol{A}$, Differences in low-frequency (2- 4 Hz) pairwise phase synchrony between correct (Corr) and incorrect (Incorr) trials between a right TPJ seed region (green) and all brain regions after cue presentation (left). ROIs exhibiting significant ( $p<0.05$, corrected for multiple comparisons) differences across participants are shown (right). $\boldsymbol{B}$, Same as $\boldsymbol{A}$ but for a left PFC seed region. $\boldsymbol{C}$, Time evolution of average differences in low-frequency $(2-4 \mathrm{~Hz}$ ) phase synchrony between the regions indicated in each subplot title. Significant temporal epochs are indicated with a red circle ( $p<0.05$, corrected for multiple comparisons in space and time). Error bars represent SEM across subjects.

presentation of word pairs that are encoded successfully in memory. These oscillatory changes begin in the right TPJ and bilateral PFC and terminate in the left temporal lobe. These patterns of cortical activation exhibit a well defined onset and offset. We found that the regions exhibiting increases in low-frequency oscillatory power were bound together through progressive lowfrequency phase synchrony that involved the MTL by the time of word presentation. An orientation cue preceding each trial triggered these changes, suggesting that the activation of this functional network may reflect attentional mechanisms that optimize memory encoding.

Our results add to a growing literature examining changes in neural activity that precede and influence episodic memory formation (Adcock et al., 2006; Otten et al., 2006; Guderian et al., 2009; Park and Rugg, 2010; Fell et al., 2011; Uncapher et al., 2011; Burianová et al., 2012). These studies have focused primarily on changes in fMRI BOLD activation (Adcock et al., 2006; Park and Rugg, 2010; Addante et al., 2015) and oscillatory changes within the hippocampus (Guderian et al., 2009; Fell et al., 2011). Our results extend these findings in several ways. First, our data build on the observed link between low-frequency hippocampal oscillations and memory (Squire et al., 2004; Manns et al., 2007; Staudigl and Hanslmayr, 2013) by demonstrating prestimulus oscillatory changes distributed across multiple cortical regions. This implicates the involvement of cognitive processes that may modulate memory formation (Sekuler and Kahana, 2007). Second, our data exploit the high temporal resolution captured by iEEG to demonstrate the specific spatiotemporal dynamics of these prestimulus changes. Third, our data show that prestimulus changes in low-frequency power are accompanied by changes in pairwise phase synchrony that correlate with successful memory encoding.

We examined all frequencies in our study, but only oscillatory power, ITPC, and phase synchrony between 2 and $4 \mathrm{~Hz}$ exhibited significant increases before successful memory encoding. Our results are consistent with previous studies suggesting that lower- 
frequency bands may modulate long-range coupling between distant areas (Liebe et al., 2012; Saalmann et al., 2012). Lowfrequency oscillations can entrain to the rhythm of behavioral tasks to optimize performance in an energy-efficient manner (Lakatos et al., 2008; Daitch et al., 2013). Because the time between presentation of the orientation cue and word pairs was $\sim 1 \mathrm{~s}$, phase-locked low-frequency activity may maximize excitability and enhance subsequent memory encoding (Lakatos et al., 2008). Whether this frequency band represents a distinct delta band is still unknown, because recent evidence suggests that human theta oscillations extends to this range (Lega et al., 2012). We did not observe significant cortical changes in prestimulus oscillatory power in the traditional theta band as has been implicated in the hippocampus (Guderian et al., 2009; Fell et al., 2011). We also found that gamma oscillatory power decreased in many regions in which we observed increased lowfrequency power and synchrony. Interestingly, this pattern of low-frequency increases and high-frequency decreases in oscillatory power is reversed after item presentation during successful encoding (Burke et al., 2013; Greenberg et al., 2015).

Our data suggest that one possible interpretation of the observed prestimulus changes in oscillatory power and network synchrony is activation of attentional networks that promote successful memory encoding (Sekuler and Kahana, 2007; Gazzaley and Nobre, 2012). During the relatively long encoding period, participants certainly have many opportunities to attend to and encode the presented word pairs. However, we note that the observed changes in prestimulus activity seen in our data and in previous studies suggest the presence of preparatory neural processes. The spatial regions in which these preparatory changes are observed overlap with anatomical locations implicated in attention (Corbetta and Shulman, 2002; Corbetta et al., 2008). Indeed, when we tested specifically whether predefined attentional regions exhibit significant differences in prestimulus spectral power, we found significant increases during correct encoding.

We observed significant increases in $2-4 \mathrm{~Hz}$ ITPC in the right TPJ and right TO region immediately after the orientation cue. Functional imaging studies have implicated the right TPJ in a ventral attention network (VAN) that preferentially responds to unexpected yet task-relevant stimuli and serves to reorient attention (Corbetta and Shulman, 2002; Corbetta et al., 2008). We found that this bottom-up attentional network was activated in our task in response to the orientation cue (Corbetta et al., 2008; Uncapher et al., 2011; Burianová et al., 2012). ITPC may represent the neural mechanism by which cognitive networks flexibly reconfigure to respond to new stimuli (Lakatos et al., 2008; Daitch et al., 2013), but we did not observe significant increases in ITPC in any other brain

$$
\text { (1) }
$$
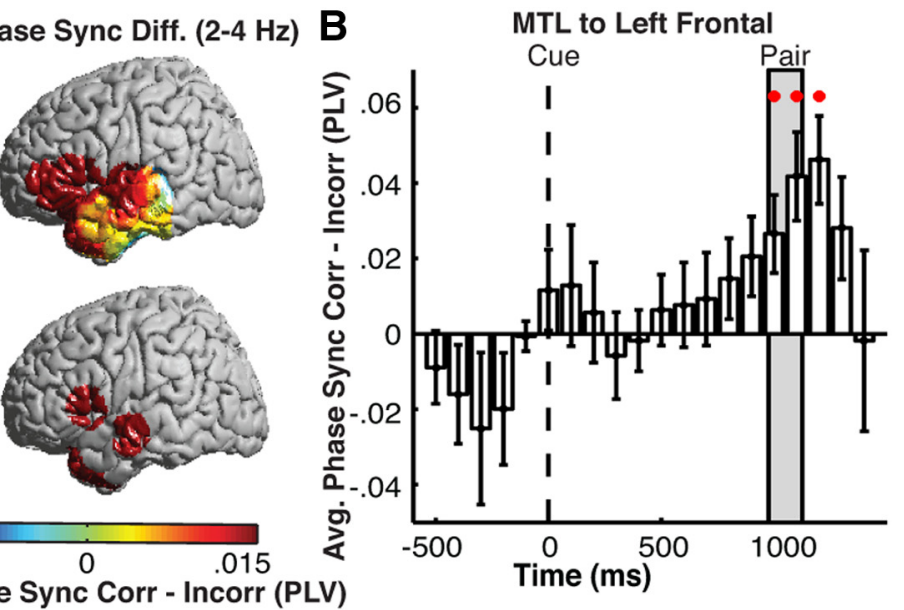

corr (PLV)

A Left MTL Phase Sync Diff. (2-4 Hz) B

Figure 6. Low-frequency pairwise phase synchrony with the left MTL. $A$, Differences in low-frequency $(2-4 \mathrm{~Hz})$ pairwise synchrony between correct (Corr) and incorrect (Incorr) trials between a left MTL seed region (green) and all brain regions (left). Time evolution of averant $(p<0.05$, corrected for multiple comparisons) differences across participants are shown (righ). $B$, left PFC. Significant temporal epochs are indicated with a red circle $(p<0.05$, corrected for multiple comparisons in space and frequency ( $2-4 \mathrm{~Hz}$ ) pairwise synchrony between the right TPJ, right frontal (RF), left PFC, left parietal (LP), and left MTL regions. Arrows are color coded with the mean peak time of increased pairwise phase synchrony. Significant increases in low-frequency ITPC are observed in right TPJ, right T0, and right temporal lobe (RT) regions.

region. In regions that are not specifically responsive to the input stimulus, changes in ITPC may be modulatory, rather than driving (Lakatos et al., 2008), and may not be evident in the macroscopic iEEG recordings. However, we cannot exclude the possibility that they occur.

In our data, the left PFC exhibited significant prestimulus increases in low-frequency power after the orientation cue and significant increases in pairwise phase synchrony with multiple cortical regions. We did not observe left PFC activation strictly in response to the task. Together, these findings suggest that initial prestimulus activation of the left PFC may play an important role in preparing cortical networks for successful memory formation. One possibility is that the left PFC is part of a larger left hemispheric dorsal attention network (DAN) that has been implicated previously in biasing cognitive resources in a top-down manner based on behavioral goals (Corbetta and Shulman, 2002; Corbetta et al., 2008). Activation of the DAN, which includes regions of the frontal lobe, parietal lobe, and frontal eye fields, may predict subsequent task performance (Sapir et al., 2005; Corbetta et al., 2008). Our data could not assess activity in the superior parietal lobe, a key region in the DAN, given the limited number of participants with electrodes in that area. Nevertheless, left frontal activation, and subsequent synchrony with and activation of 
the temporal lobe, suggests the possible involvement of a topdown network during successful encoding.

The presentation of the behaviorally relevant orientation cue may in fact activate both networks (Corbetta et al., 2008). Like the right TPJ, we also observe activation of the left PFC in response to the bottom-up orientation cue. In addition, a component of the activity observed here involves the right PFC, which has been noted as a possible link between the VAN and DAN (Fox et al., 2006). Importantly, we found that the PFC of both networks were bound through synchronous lowfrequency oscillations, providing a possible mechanism by which the two networks may communicate. Indeed, previous evidence has demonstrated that the two networks are connected functionally (Uncapher et al., 2011; Burianová et al., 2012; Daitch et al., 2013).

How such activity bridges the gap between attention and memory before item encoding remains unclear (Cabeza et al., 2008). Top-down visual attention may increase the firing rate of neurons in goal-relevant areas, mediate communication between cortical regions by increasing synchrony, reduce extraneous noise, or amplify neural representations of certain features (Fries et al., 2001; Buschman and Miller, 2007; Baldauf and Desimone, 2014). The diversion of attentional resources to enhance memory may operate in a similar way as its sensory counterparts (Gazzaley and Nobre, 2012). Therefore, top-down attention may prepare the hippocampus for specific encoding processes (Uncapher and Rugg, 2009). Our results support this hypothesis in a number of ways. The functional connections we observed here progressed from core regions of the VAN to regions of the left hemispheric DAN and ultimately terminated in the temporal lobe and MTL structures. Oscillatory coupling between the cortex and hippocampus has been identified previously with successful encoding (Fell et al., 2001; Ekstrom and Watrous, 2014), and so the synchrony we observed at the time of word presentation may reflect the final step in communication between attentional and encoding networks.

Together, our data suggest that prestimulus low-frequency oscillatory power and phase synchrony are coordinated across multiple cortical regions to promote memory encoding. How well participants attend to the unexpected cue in our task in large part determines to what extent these networks are activated and hence how well they encode subsequent associations. In the absence of such a bottom-up stimulus, it is possible that attentional network activation would not be as robust. Therefore, our data provide a link between attentional processes and episodic memory formation and suggest that low-frequency cortical oscillatory power and functional connectivity may be the neural mechanism through which this link occurs. Hence, our data provide insight into how prestimulus cortical network activity may optimize functional brain states in preparation for successful encoding.

\section{References}

Adcock RA, Thangavel A, Whitfield-Gabrieli S, Knutson B, Gabrieli JD (2006) Reward-motivated learning: mesolimbic activation precedes memory formation. Neuron 50:507-517. CrossRef Medline

Addante RJ, de Chastelaine M, Rugg MD (2015) Pre-stimulus neural activity predicts successful encoding of inter-item associations. Neuroimage 105:21-31. CrossRef Medline

Baldauf D, Desimone R (2014) Neural mechanisms of object-based attention. Science 344:424-427. CrossRef Medline

Burianová H, Ciaramelli E, Grady CL, Moscovitch M (2012) Top-down and bottom-up attention-to-memory: mapping functional connectivity in two distinct networks that underlie cued and uncued recognition memory. Neuroimage 63:1343-1352. CrossRef Medline

Burke JF, Zaghloul KA, Jacobs J, Williams RB, Sperling MR, Sharan AD, Kahana MJ (2013) Synchronous and asynchronous theta and gamma activity during episodic memory formation. J Neurosci 33:292-304. CrossRef Medline

Buschman TJ, Miller EK (2007) Top-down versus bottom-up control of attention in the prefrontal and posterior parietal cortices. Science 315: 1860-1862. CrossRef Medline

Cabeza R, Ciaramelli E, Olson IR, Moscovitch M (2008) The parietal cortex and episodic memory: an attentional account. Nat Rev Neurosci 9:613625. CrossRef Medline

Corbetta M, Shulman GL (2002) Control of goal-directed and stimulus-driven attention in the brain. Nat Rev Neurosci 3:201-215. CrossRef Medline

Corbetta M, Patel G, Shulman GL (2008) The reorienting system of the human brain: from environment to theory of mind. Neuron 58:306-324. CrossRef Medline

Daitch AL, Sharma M, Roland JL, Astafiev SV, Bundy DT, Gaona CM, Snyder AZ, Shulman GL, Leuthardt EC, Corbetta M (2013) Frequency-specific mechanism links human brain networks for spatial attention. Proc Natl Acad Sci U S A 110:19585-19590. CrossRef Medline

Dykstra AR, Chan AM, Quinn BT, Zepeda R, Keller CJ, Cormier J, Madsen JR, Eskandar EN, Cash SS (2012) Individualized localization and cortical surface-based registration of intracranial electrodes. Neuroimage 59: 3563-3570. CrossRef Medline

Ekstrom AD, Watrous AJ (2014) Multifaceted roles for low-frequency oscillations in bottom-up and top-down processing during navigation and memory. Neuroimage 85:667-677. CrossRef Medline

Fell J, Klaver P, Lehnertz K, Grunwald T, Schaller C, Elger CE, Fernández G (2001) Human memory formation is accompanied by rhinal-hippocampal coupling and decoupling. Nat Neurosci 4:1259-1264. CrossRef Medline

Fell J, Ludowig E, Staresina BP, Wagner T, Kranz T, Elger CE, Axmacher N (2011) Medial temporal theta/alpha power enhancement precedes successful memory encoding: evidence based on intracranial EEG. J Neurosci 31:5392-5397. CrossRef Medline

Fox MD, Corbetta M, Snyder AZ, Vincent JL, Raichle ME (2006) Spontaneous neuronal activity distinguishes human dorsal and ventral attention systems. Proc Natl Acad Sci U S A 103:10046-10051. CrossRef Medline

Fries P, Reynolds JH, Rorie AE, Desimone R (2001) Modulation of oscillatory neuronal synchronization by selective visual attention. Science 291: 1560-1563. CrossRef Medline

Gazzaley A, Nobre AC (2012) Top-down modulation: bridging selective attention and working memory. Trends Cogn Sci 16:129-135. CrossRef Medline

Greenberg JA, Burke JF, Haque R, Kahana MJ, Zaghloul KA (2015) Decreases in theta and increases in high frequency activity underlie associative memory encoding. Neuroimage 114:257-263. CrossRef Medline

Gruber MJ, Otten LJ (2010) Voluntary control over prestimulus activity related to encoding. J Neurosci 30:9793-9800. CrossRef Medline

Guderian S, Schott BH, Richardson-Klavehn A, Düzel E (2009) Medial temporal theta state before an event predicts episodic encoding success in humans. Proc Natl Acad Sci U S A 106:5365-5370. CrossRef Medline

Haynes JD, Sakai K, Rees G, Gilbert S, Frith C, Passingham RE (2007) Reading hidden intentions in the human brain. Curr Biol 17:323-328. CrossRef Medline

Lachaux JP, Rodriguez E, Martinerie J, Varela FJ (1999) Measuring phase synchrony in brain signals. Hum Brain Mapp 8:194-208. CrossRef Medline

Lakatos P, Karmos G, Mehta AD, Ulbert I, Schroeder CE (2008) Entrainment of neuronal oscillations as a mechanism of attentional selection. Science 320:110-113. CrossRef Medline

Lega BC, Jacobs J, Kahana M (2012) Human hippocampal theta oscillations and the formation of episodic memories. Hippocampus 22:748-761. CrossRef Medline

Leube DT, Erb M, Grodd W, Bartels M, Kircher TT (2001) Differential activation in parahippocampal and prefrontal cortex during word and face encoding tasks. Neuroreport 12:2773-2777. CrossRef Medline

Liebe S, Hoerzer GM, Logothetis NK, Rainer G (2012) Theta coupling between $\mathrm{v} 4$ and prefrontal cortex predicts visual short-term memory performance. Nat Neurosci 15:456-462, S1-S2. CrossRef Medline

Linkenkaer-Hansen K, Nikulin VV, Palva S, Ilmoniemi RJ, Palva JM (2004) Prestimulus oscillations enhance psychophysical performance in humans. J Neurosci 24:10186-10190. CrossRef Medline

Manns JR, Howard MW, Eichenbaum H (2007) Gradual changes in hip- 
pocampal activity support remembering the order of events. Neuron 56 : 530-540. CrossRef Medline

Maris E, Oostenveld R (2007) Nonparametric statistical testing of EEG- and MEG-data. J Neurosci Methods 164:177-190. CrossRef Medline

Otten LJ, Quayle AH, Akram S, Ditewig TA, Rugg MD (2006) Brain activity before an event predicts later recollection. Nat Neurosci 9:489-491. CrossRef Medline

Park H, Rugg MD (2010) Prestimulus hippocampal activity predicts later recollection. Hippocampus 20:24-28. CrossRef Medline

Saalmann YB, Pinsk MA, Wang L, Li X, Kastner S (2012) The pulvinar regulates information transmission between cortical areas based on attention demands. Science 337:753-756. CrossRef Medline

Sapir A, d'Avossa G, McAvoy M, Shulman GL, Corbetta M (2005) Brain signals for spatial attention predict performance in a motion discrimination task. Proc Natl Acad Sci U S A 102:17810-17815. CrossRef Medline

Sekuler R, Kahana MJ (2007) A stimulus-oriented approach to memory. Curr Dir Psychol Sci 16:305-310. CrossRef Medline

Smith EE, Jonides J (1999) Storage and executive processes in the frontal lobes. Science 283:1657-1661. CrossRef Medline
Squire LR, Clark RE, Bayley PJ (2004) The cognitive neurosciences. Cambridge, MA: Massachusetts Institute of Technology.

Staudigl T, Hanslmayr S (2013) Theta oscillations at encoding mediate the contex-dependent nature of human episodic memory. Curr Biol 23: 1101-1106. CrossRef Medline

Staudigl T, Vollmar C, Noachtar S, Hanslmayr S (2015) Temporal-pattern similarity analysis reveals the beneficial and detrimental effects of context reinstatement on human memory. J Neurosci 35:5373-5384. CrossRef Medline

Uncapher MR, Rugg MD (2009) Selecting for memory? the influence of selective attention on the mnemonic binding of contextual information. J Neurosci 29:8270-8279. CrossRef Medline

Uncapher MR, Hutchinson JB, Wagner AD (2011) Dissociable effects of top-down and bottom-up attention during episodic encoding. J Neurosci 31:12613-12628. CrossRef Medline

Yaffe RB, Kerr MS, Damera S, Sarma SV, Inati SK, Zaghloul KA (2014) Reinstatement of distributed cortical oscillations occurs with precise spatiotemporal dynamics during successful memory retrieval. Proc Natl Acad Sci U S A 111:18727-18732. CrossRef Medline 